See discussions, stats, and author profiles for this publication at: https://www.researchgate.net/publication/321253022

\title{
Multiscaling properties on sequences of turbulent plumes images
}

Article in Chaos Solitons \& Fractals · December 2017

DOI: 10.1016/j.chaos.2017.10.011

CITATIONS

5

5 authors, including:

\section{Pilar López}

Complutense University of Madrid

58 PUBLICATIONS 96 CITATIONS

SEE PROFILE

Ania Matulka

Puertos del Estado

33 PUBlications 131 CITATIONS

SEE PROFILE
READS

290

Ana M. Tarquis

Universidad Politécnica de Madrid

368 PUBLICATIONS 2,503 CITATIONS

SEE PROFILE

Jose Manuel Redondo

Universitat Politècnica de Catalunya

317 PUBlicATIONS 1,803 CITATIONS

SEE PROFILE

Some of the authors of this publication are also working on these related projects:

Agro-Environmental Sustainability View project

Drought/Flood Remote Sensing View project 


\title{
Multiscaling properties on sequences of turbulent plumes images
}

\author{
Pilar López ${ }^{\mathrm{a}, *}$, Ana M. Tarquis ${ }^{\mathrm{b}}$, Ania Matulka ${ }^{\mathrm{c}}$, Benjamin Skadden ${ }^{\mathrm{b}}$, José M. Redondo ${ }^{\mathrm{d}}$ \\ a Department of Applied Mathematics (Biomathematics), University Complutense of Madrid, Madrid 28040, Spain \\ ${ }^{\mathrm{b}}$ Research and Studies Center for the Management of Agricultural and Environmental Risks (CEIGRAM), University Politécnica of Madrid, Madrid 28040, \\ Spain \\ 'Center for Marine Environmental Sciences (MARUM), Department of Geosciences, Geosystem Modeling, Bremen 28359, Germany \\ d Department of Applied Physics, University Politécnica of Cataluña, Barcelona 08034, Spain
}

\section{A R T I C L E I N F O}

Article history:

Received 13 July 2017

Revised 5 October 2017

Accepted 10 October 2017

\section{Keywords:}

Multifractal method

Multifractal spectrum

Turbulent plumes

Entrainment coefficient

Spectrum symmetry

\begin{abstract}
A B S T R A C T
A multifractal analysis on a finite-range-scale of the plume concentration images at different experimental conditions (the height of the source $H_{0}$ ), where the measure is the grey value of the image (from 0 to 255), was applied to study its structure through time. The multifractal spectrum showed the characteristic inverse U-shape and a similar evolution in all $H_{0}$. The variation of the Hölder exponent $(\Delta \alpha)$ presented different amplitudes at different moments and increased with time. The symmetry of the spectrum $(\Delta f)$ decreased with time achieving negative values (from left hand asymmetry evolving to right asymmetry). We show the different behaviour of axial velocity $(W)$ with $\Delta \alpha$ and $\Delta f$. There is a linear relation of entrainment coefficient $\left(\alpha_{e}\right)$ and the entropy dimension $\left(\alpha_{1}\right)$. Therefore, the multifractal spectrum and the derived parameters can be used as markers of plume evolution as well as to study the effect of experimental conditions.
\end{abstract}

(c) 2017 Elsevier Ltd. All rights reserved.

\section{Introduction}

Turbulent plumes are fluid motions whose primary sources of kinetic energy and momentum flux are body forces derived from density differences [1,2]. The plume boundary is an edge across which the ambient fluid is entrained and the plume boundary moves at the velocity of the plume fluid. In geophysics, it is usually the generation of turbulent plumes as a part of a dispersion process. For example, volcanic plumes or river plumes can be observed where a stream, usually a river, empties into a lake, sea or ocean. Therefore, the geophysical importance of turbulent plumes is clear. It is very interesting to use fractal methods to analyse satellite images to detect and quantify the time behaviour of volcanic plumes to study problems related to environmental impacts or aviation hazards [3,4]. It is also possible to determine the direction of littoral transport at a time if we study the geometry of a river plume. Fractal methods can do this analysis [5]. Largerscale features of plumes are generally well-represented by the fractal method characterizing scalar isosurfaces in terms of fractal and multifractal properties. Many other processes can be adequately described by fractals, such as river networks [6], rainfall dynamics [7], cloud shapes [8] and turbulent dispersion of a contaminant in the atmospheric boundary layer [9-12].

\footnotetext{
* Corresponding author

E-mail address: maplopez@bio.ucm.es (P. López).
}

The fractal technique has been used to analyse turbulent fields in several contexts and provides a natural method for describing the self-similar nature of processes [13]. These are useful tools to analyse the geometric evolution of surfaces in turbulent flows and the implications of this geometric behaviour on mixing [14].

Several studies analyse the relation between the fractal dimension of various surfaces (boundary layers, axisymmetric jets, plane wakes and mixing layers) in high Reynolds number turbulent flows. In 1989, Sreenivasan et al, summarized the previous results on the fractal dimensions of scalar and vorticity interfaces in several classical turbulent flows (a fractal dimension of $2.35 \pm 0.05$ [15-16]). Fractal dimensions between 1.3 and 1.35 are obtained from LES (large-eddy simulation) plumes for neutral and convective conditions [17]. Prasad and Sreenivasan used the box-counting method to analyse images of jet sections and determined that the fractal dimension of jet boundaries was 1.36 , which is close to estimates from atmospheric data [14]. Hentschel and Procaccia predicted a slightly higher cloud perimeter fractal dimension in the range between 1.37 and 1.41 [13]. Sykes and Gabruk determined a fractal dimension (roughly 1.30-1.35) for the scalar concentration field of a turbulent plume dispersion [17]. Lane-Serff investigated the effects of buoyancy on the fractal structure of round, turbulent jets and plumes. He also measured the fractal dimension of concentration contours in jets and plumes, which had an apparent minimum of 1.23 [18]. 
Many processes are highly intermittent with spiky measures and strong nonuniformities (for example, the distribution of a turbulent kinetic energy dissipation rate). These intermittent processes cannot be well-described by the typical moment methods and, therefore, a multifractal method is required [19]. Multifractal analysis aims to deduce the multifractal spectrum which is measured for several positive magnitudes that characterize small-scale motions [20-22]. The multifractal description is more general than other theories. Some of these are special cases; for example, the point $f(\alpha=1)=1$ in the energy dissipation rate spectrum is Kolmogorov's theory (K41). Actually, we consider that turbulence is a multiplicative process and, therefore, the multifractal method can be used to study turbulence [12]. Puthenveettil et al. conducted the multifractal analysis using binary images and the standard box counting methodology to estimate the multifractal exponents [23]. Finally, Lane-Serff concluded that the use of a single value for the fractal dimension in jets and plumes is questionable [18].

The objective of our study is to apply a multifractal analysis to compare the characteristics of the multifractal spectrum, obtained from grey-scale images of the plume at different times and under different initial conditions, with the classical magnitudes that characterize the plume's dynamic. First, we describe the experimental procedure to generate a turbulent plume, its main characteristics and the multifractal method in Section 2. In $\S 3$, we present the multifractal results and their comparison with the axial velocity and the entrainment coefficient of the plume. Finally, in $\S 4$ we present the conclusions and we discuss the relevance of this analysis for the presented case study.

\section{Material and methods}

\subsection{Experimental setup and procedures}

The aim of the experimental procedure is to generate a turbulent axysimmetric plume, controlling its position and its physical characteristics as buoyancy and momentum fluxes. We release a volume fluid vertically down (with a flow rate up to $8.40 \mathrm{~cm}^{3} \mathrm{~s}^{-1}$ ) from a small orifice, with a diameter $d=0.6 \mathrm{~cm}$, into a stationary body of water with a height of $16.5 \mathrm{~cm}$ contained in a glass tank of dimensions $32 \mathrm{~cm}$ high and a $25 \mathrm{~cm} \times 25 \mathrm{~cm}$ cross-section. The small orifice is located at a height $H_{0}$ which takes values of $2 \mathrm{~cm}$, $3 \mathrm{~cm}, 3.5 \mathrm{~cm}$ and $6.5 \mathrm{~cm}$ and, therefore, increases the overall initial potential energy of the fluid system and the momentum flux. The Atwood number, $A$, measures the density difference of the fluid system. The Reynolds number at the source, based on the source diameter and the mean velocity there, is approximately 2000 . The flow was not observed in the far field (ranging between 250-d and $550-d$ ) due to the dimensions of the tank. The releasing fluid was a potassium permanganate solution $\left(500 \mathrm{~cm}^{3}\right)$ which is considered as incompressible and miscible and, as such, presents a high Schmidt number (of the order of $10^{3}$ ) and has an intense purple colour (from pink to mauve). Thus, it was not necessary to add a dye as passive tracer and the flow was directly visualized. A detailed description of the experimental setup can be found in López [24] and in López, Cano and Redondo [25].

The flow was back illuminated from conventional fluorescent lights approximately $0.5 \mathrm{~m}$ from the tank giving a projection. This procedure gives an integral image of the plume volume and the registered images average the concentration over the plume volume. The flow was recorded by a high-quality digital video system at high velocity mode (100 fps). The video recordings of the experiments were sequenced into frames using a frame-sequencer software (VirtualDubMod). The frame array had a resolution of $640 \times 480$ pixels capturing the area of $25 \times 18 \mathrm{~cm}^{2}$. Each frame has intensities recorded as integers in ranging from $0-255$. For each experimental video, 288 frames were obtained and between 40 and 60 frames of the time-dependent, three-dimensional plume dispersion were used for the multifractal analysis (those without interaction of the plume with the tank contours).

Fig. 1 shows a sequence of digitized video images from a single experiment and show the time evolution of a turbulent plume. Upon entering the ambient fluid, the source fluid becomes unstable and forms a turbulent plume at the centre of the tank (Fig. 1(a)(d)). As the plume is gravitationally unstable, it engulfs lighter fluid as it evolves and there is entrainment of the ambient fluid that is directed through the border of the turbulent plume [26]. The downward speed of the plume produces an upward recirculating movement in the ambient fluid which favours the mixing between them.

The behaviour of turbulent plumes is described by three ordinary differential equations for the fluxes of volume, momentum and buoyancy under the Boussinesq assumption [1]. The governing parameters are the radius $r$, the vertical velocity $W$, the entrainment velocity $U_{e}$ and the reduced gravity $g$ '.

The difference between the plume -fluid radial velocity and the total fluid velocity naturally quantifies the purely horizontal entrainment flux of ambient fluid into the plume. This process is characterized by an inflow speed perpendicular to the plume axis which is characterized by the entrainment assumption [1,26-28]. This hypothesis states that the rate of transfer of ambient fluid into the plume, $U_{E}$, is proportional to the mean centre-line vertical speed of the plume, $W$, or axial velocity. The ratio of inflow or entrainment velocity to the plume vertical speed is called the entrainment constant: $\alpha_{E}=U_{E} / W$. Fig. 2 shows these main magnitudes that characterize the dynamics of a plume overwritten on the third frame of Fig. $1(t=0.44 \mathrm{~s})$.

Away from the exit of the nozzle, similarity arguments show that the plume spreads linearly and the axial velocity decreases inversely to the distance. The mean flow model described by Morton et al. gives the plume radius $r$ proportional to the distance from the source $z\left(r=6 \alpha_{P} z / 5\right)$ where $\alpha_{p}$ is the entrainment coefficient for a plume and the mean vertical speed $W$ is proportional to $r^{-3}$ $\left(W \propto r^{-3}\right)$ for plumes [1,18].

Images of plumes, such as other digital imagery, typically contain a large proportion of mixed-pixels (pixels whose digital number is the weighted average of more than one constituent, such as a water/sodium permanganate). To facilitate identification of constituent peaks in the grey-scale histogram, a 2-D filter, executed in NIH Image [29], was run on each frame to mask pixels which differed by more than $0.1 \%$ from the surrounding neighbourhood of 25 pixels $(5 \times 5$ unit area). Full details of this technique can be found in Elliot and Heck [30]. The resulting images are shown in Fig. 1(e)-(h).

\subsection{Multifractal analysis}

Multifractal techniques divide the full image of analysis into boxes to construct samples at different scales. The size of the box for implementing the multifractal method will be the one between the highest resolutions ( 1 pixel) to the highest size of the full image. A partitioning process starting from the smallest resolution to successively form larger boxes combining pixels is called upscaling process. There are two main methods of upscaling process: the box counting method and gliding box method. In this study, we have applied the latter.

In box counting method the number of boxes will be smaller when the box size $\varepsilon$ goes closer to 1 . This implies that the number of samples will not be enough for carrying out good statistical analysis, increasing the error associated to the measure. On the contrary, gliding box method construct samples gliding a box over the grid map in all possible ways provided that the box is completely bounded by the grid map. Through this procedure more 

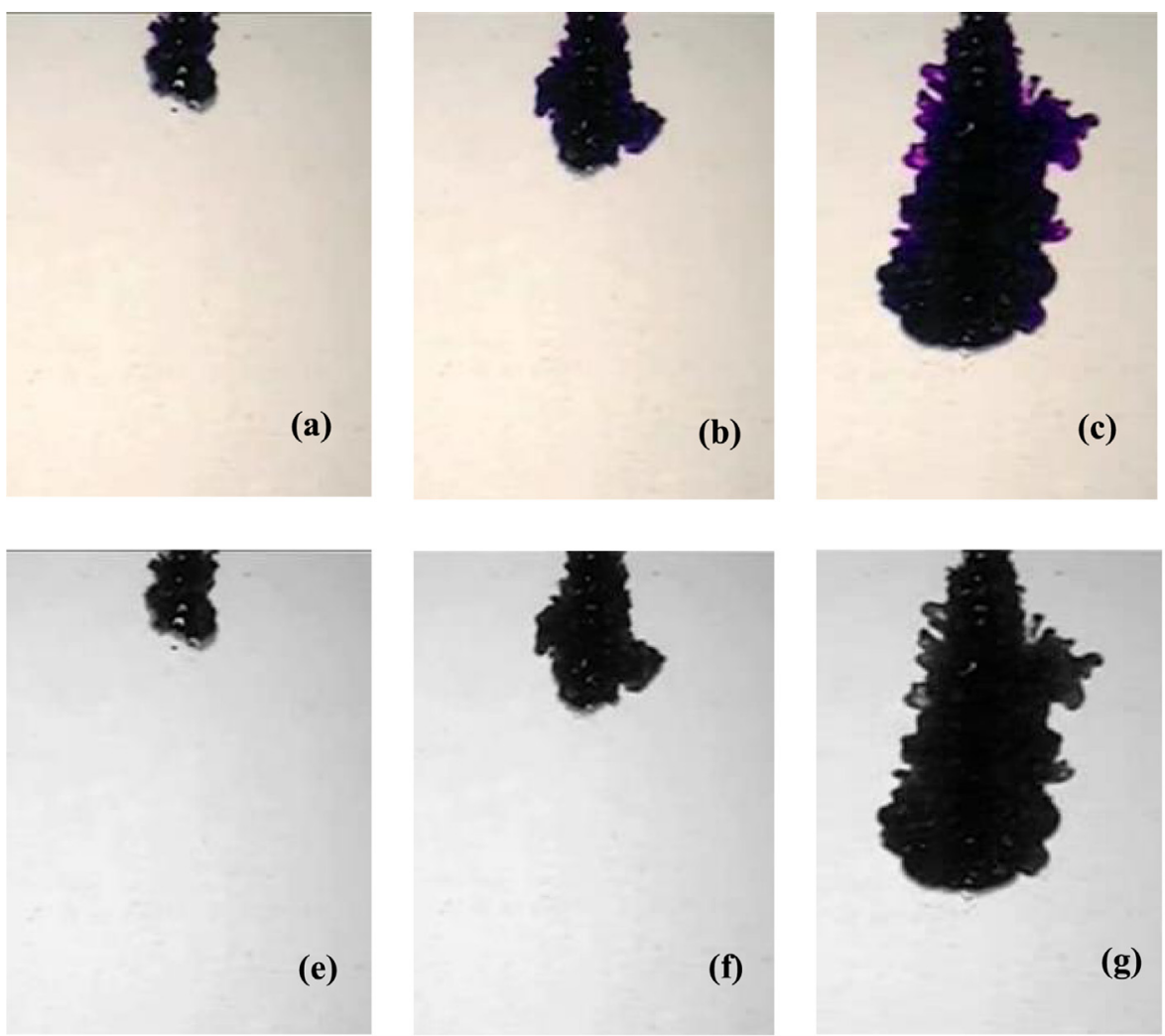

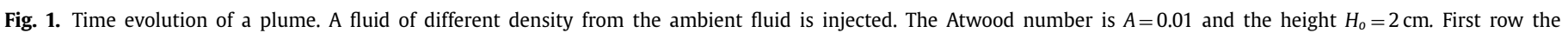
original images and second row the grey images for the following times: (a) and (e) $0.03 \mathrm{~s}$; (b) and (f) $0.15 \mathrm{~s}$; (c) and (g) $0.44 \mathrm{~s}$; (d) and (h) $0.59 \mathrm{~s}$.

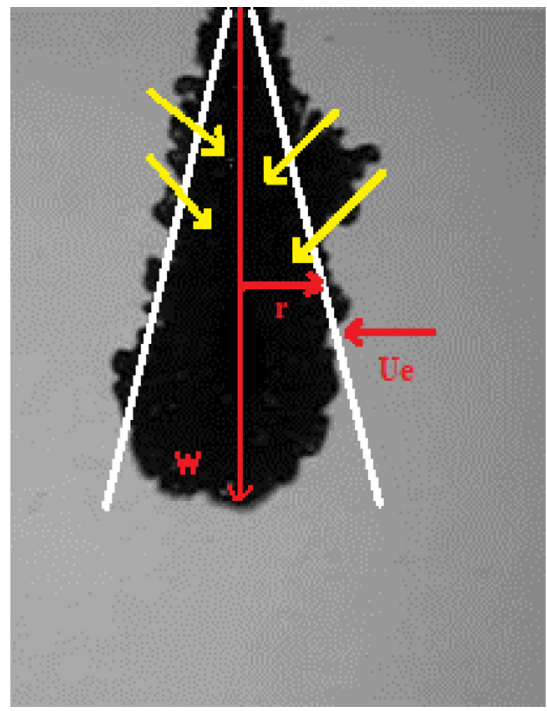

Fig. 2. The main magnitudes of a turbulent plume. The axial vertical velocity is $W, r$ is the plume radius and $U_{e}$ is the radial entrainment velocity at which the ambient fluid is come into the plume. The tilted arrows show the direction of the engulfed ambient fluid.

samples are obtained for each scale, improving the statistical significance and reducing the measurement uncertainty [31].

The gliding box method was originally used in lacunarity analysis [32] and later modified by Cheng [33] for application to multifractal analysis and then used in soil analysis. First estimates the mass exponent function $\tau(q)$ where $q$ is the statistical moments order:

$\tau(q)=\frac{\log \left(\frac{1}{N(\varepsilon)} \sum_{i=1}^{N(\varepsilon)} \mu_{i}^{q}(\varepsilon)\right)}{\log \varepsilon}$

where $\varepsilon$ is the dimensionless box size, $N(\varepsilon)$ represents total number of gliding boxes of size $\varepsilon$ with measure $\mu(\varepsilon) \neq 0$ where $\mu$ represents the grey value of the image (from 0 to 255). Finally, $E$ is the topological dimension of the object ( $E=2$ for two-dimensional objects and $E=3$ for three dimensional objects).

The singularity index $\alpha$, or Hölder exponent, can be determined by the Legendre transformation of the $\tau(q)$ curve as [34]

$\alpha=\frac{d \tau(q)}{d q}$.

If $q=0$, the coefficient $\alpha$ is the fractal dimension. The exponent $\alpha$ is called the entropy dimension when $q=1$ and, finally, if $q=2, \alpha$ represents the correlation dimension.

The number of cells of size $\varepsilon$ with the same $\alpha, N_{\alpha}(\varepsilon)$, is related to the cell size as $N_{\alpha}(\varepsilon) \propto \varepsilon^{-f(\alpha)}$, where $f(\alpha)$ is a scaling exponent of the cells with common $\alpha$. This parameter $f(\alpha)$ can be estimated numerically as [34]:

$f(\alpha)=q \alpha(q)-\tau(q)$.

Assuming that the uncertainty measurement is propagated proportional to the one associated for $\tau(q)$ which is calculated analytically. So the uncertainty of $\alpha$ will be:

$\Delta \alpha=\frac{\Delta \tau}{\tau} \alpha$.

In the case that the variable under study behaves as a multifractal measure, the shape of the multifractal spectrum (MFS), i.e., a graph of $f(\alpha)$ vs. $\alpha$, will be concave down parabolic shape (Fig. 3). From the MFS several parameters can be calculated to quantify its shape 


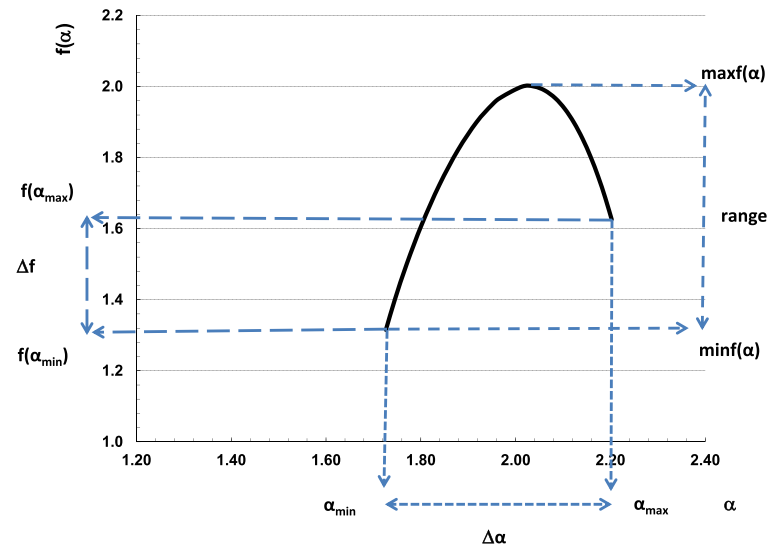

Fig. 3. Multifractal spectrum (MFS) example. We show the parameters derived from it to quantify its shape. The minimum point $\left(\alpha_{\min }, f\left(\alpha_{\min }\right)\right)$ corresponds in this case to $q=+10$ and the maximum point $\left(\alpha_{\max }, f\left(\alpha_{\max }\right)\right)$ corresponds to $q=-10$.

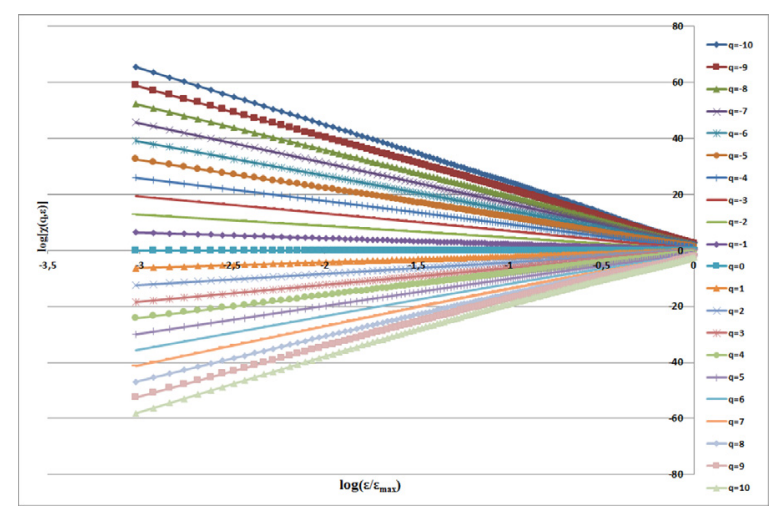

Fig. 4. The partition function $\chi(q, \varepsilon)$ versus the length size of the box $\varepsilon$. Each colour represents the different values of $q$. Frame time is $1.02 \mathrm{~s}$. Experimental conditions: $A=0.01$ and $H_{0}=2 \mathrm{~cm}$. (For interpretation of the references to colour in this figure legend, the reader is referred to the web version of this article.)

as it is described in Fig. 3. The asymmetry of MFS $(\Delta f)$ to the right and left indicates scaling domination of small and large values, respectively [35]. The width of the MFS on singularity indexes $(\Delta \alpha)$ as in the $f(\alpha)$ values (range) indicates overall complexity [36,37].

The Hausdorff dimension calculated for $q=1\left(\alpha_{1}=\alpha(q=1)=\right.$ $f(q=1))$ corresponds to the entropy dimension of the distribution related to the information or Shannon entropy [38], and quantifies the degree of disorder present in a distribution. According to Andraud et al. [39] and Gouyet [40] a $\alpha_{1}$ value close to 1.0 characterizes a system uniformly distributed throughout all scales, whereas a $\alpha_{1}$ close to 0 reflects a subset of the scale in which the irregularities are concentrated.

\section{Results and discussion}

\subsection{Multifractal analysis results}

First, we calculate the partition function $\chi(q, \varepsilon)$ where $\varepsilon$ is the length size of the box. This function represents the qth-order moment of the probability distribution of the grey values [34]. Fig. 4 shows the partition function versus $\varepsilon$ on a log-log scale for different values of $q$ ranging from -10 to 10 with by increments of 1 . All the curves show a high degree of linearity, which means that there is a relation between scales. However, this similar linear behaviour depends on the sign of $q$. If $q$ is positive, the lines are decreasing and if $q$ is negative, we have growing straight lines. All these characteristics note the multifractality of the measure (the grey value).

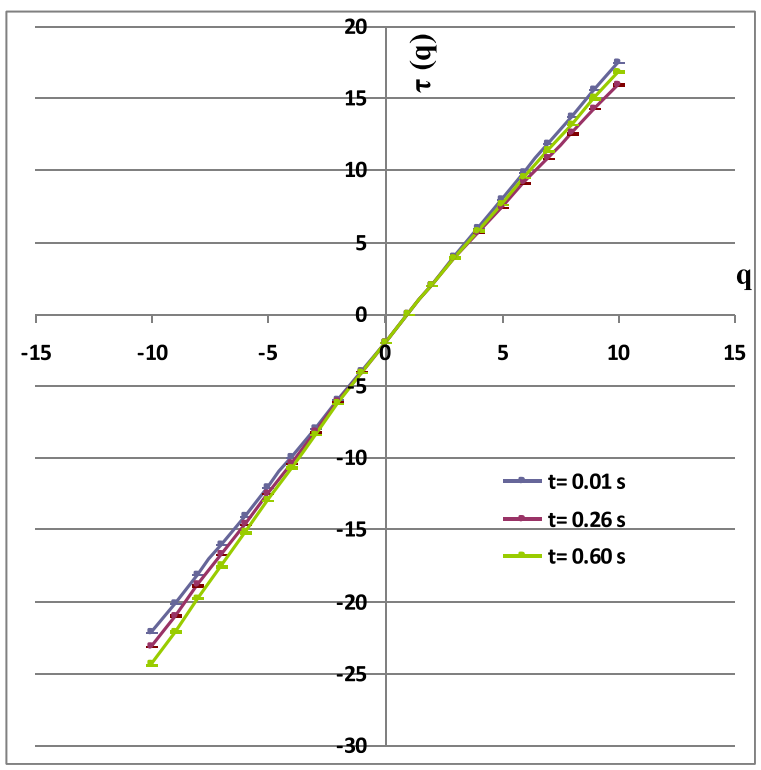

Fig. 5. The mass exponent function $\tau(q)$ versus $q$. Each colour represents the frame time of the experiment with conditions: $A=0.01$ and $H_{0}=2 \mathrm{~cm}$. (For interpretation of the references to colour in this figure legend, the reader is referred to the web version of this article.)

From these straight lines, we obtain their slopes, which are the corresponding values of the mass exponent function $\tau(q)$. For any given sample, there is a smooth variation with $q$.

Second, the mass exponent function $\tau(q)$ is calculated for different time frames and different experiments to analyse the multifractal tendency. Fig. 5 shows the mass exponent function $\tau(q)$ versus $q$ for different times of one experiment (with $A=0.01$ and $\mathrm{Ho}=2 \mathrm{~cm}$ ). All curves show some degree of nonlinearity and have different $\tau(q)$ behaviour. This expresses scale dependence, a multifractal tendency and time dependence [36]. The null value for $\tau(q)$ is reached when $q$ is 1 due to the conservative character of this measure.

Finally, the graph of the Hölder exponent $\alpha$ versus $f(\alpha)$ (multifractal spectrum) for different times and different experiments is calculated. The multifractal spectrum quantitatively characterizes variability of the measure (grey values from 0 to 255) studied with asymmetry to the right and left, indicating domination of small and large values, respectively [35]. The multifractal analysis on each grey image revealed the multiscaling nature from 1 to 196 pixels side length of the gliding box. Our study is based on this range of scales.

Additionally, in all the MFS, the dimension of the support of the measure is $2(f(\alpha)$ for $q=0)$, the dimension of an Euclidean plane. This case shows a multifractal measure where its support is not fractal [41].

The multifractal spectrum shown at three times for different experimental conditions remark a common behaviour in their evolution. Fig. 6 shows the function $f(\alpha)$ versus $\alpha$ for different times of several selected experiments with different heights $\left(H_{0}\right)$.

At the beginning (blue line), the spectrum width is narrow and presents a left hand asymmetry pointing out a narrow range of $\alpha$ values and a strong scaling behaviour in high grey values (lighter pixels). In a second moment (grey line), the spectrum becomes wider and keeps the left asymmetry with a different strength depending on the $H_{o}$ of the experiment. Later, (red line) the spectrum becomes more symmetrical and wider. This indicates the same scaling strength in the lower grey values as in higher ones. However, depending on the experimental $H_{O}$ a slight right asymme- 

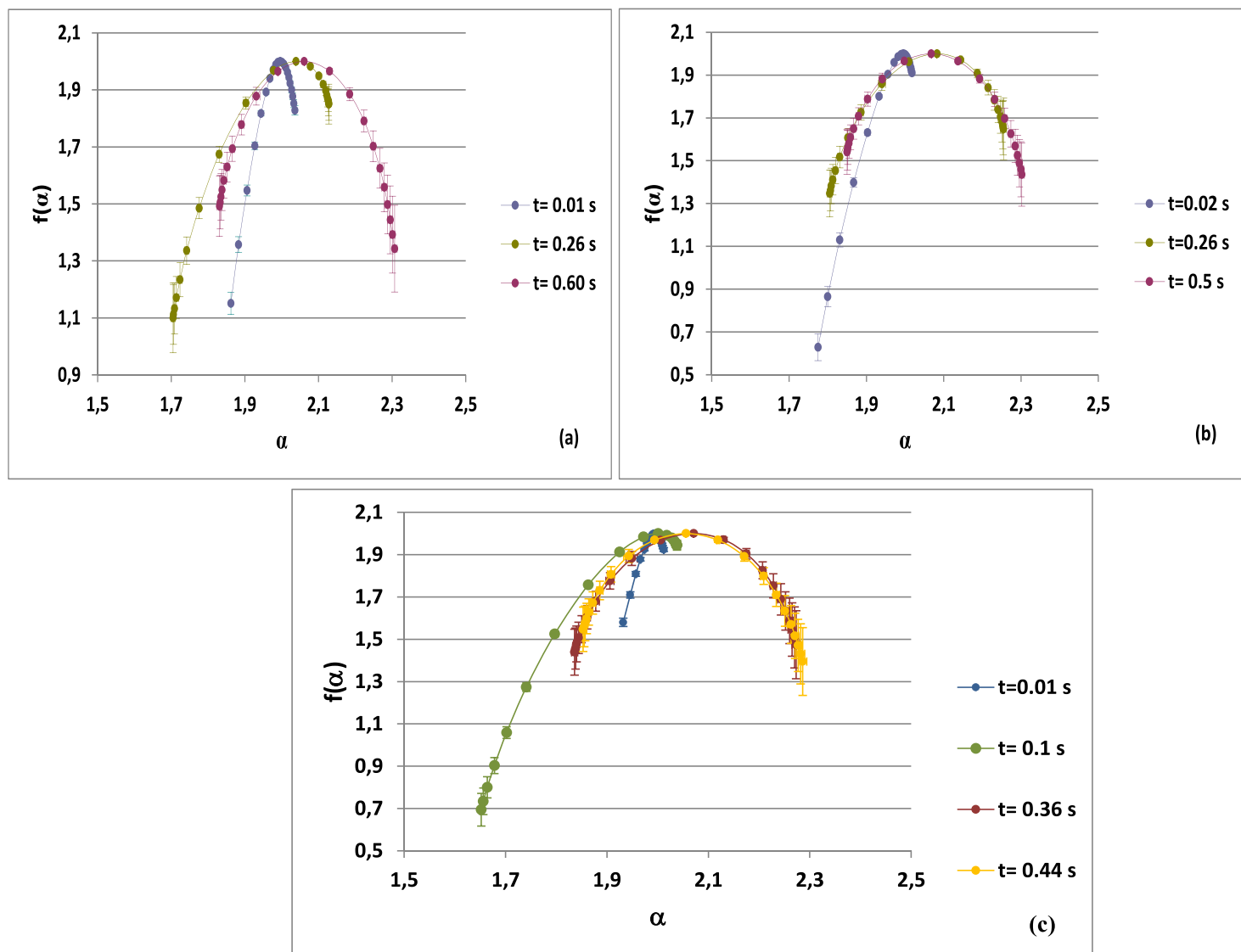

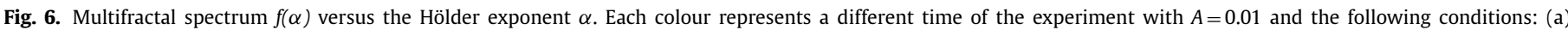
$H_{0}=2 \mathrm{~cm}$; (b) $H_{0}=3.5 \mathrm{~cm}$; (c) $H_{0}=6.5 \mathrm{~cm}$. (For interpretation of the references to colour in this figure legend, the reader is referred to the web version of this article.)

Table 1

Parameters extracted from the multifractal spectrum based on the grey values measure at different times $t$ for experiments with the Atwood number $A=0.01$. Hölder exponent at $q=-10\left(\alpha_{\max }\right), q=1\left(\alpha_{1}\right), q=10\left(\alpha_{\min }\right)$, and $\Delta \alpha=\alpha_{\max }-\alpha_{\min }$. Multifractal value at $\alpha_{\max }$ is $f\left(\alpha_{\max }\right)$, at $\alpha_{\min }$ is $f\left(\alpha_{\min }\right)$ and $\Delta f=f\left(\alpha_{\max }\right)-f\left(\alpha_{\min }\right)$.

\begin{tabular}{|c|c|c|c|c|c|c|c|c|}
\hline $\mathrm{Ho}(\mathrm{cm})$ & $t(s)$ & $\alpha_{\min }$ & $\alpha_{1}$ & $\alpha_{\max }$ & $\Delta \alpha$ & $f\left(\alpha_{\min }\right)$ & $f\left(\alpha_{\max }\right)$ & $\Delta f$ \\
\hline $2.0 \pm 0.1$ & $0.010 \pm 0.01$ & $1.862 \pm 2.0010^{-3}$ & $1.990 \pm 0.0010^{\circ}$ & $2.036 \pm 7.7210^{-4}$ & $0.174 \pm 0.003$ & $1.151 \pm 0.039$ & $1.828 \pm 0.016$ & $0.677 \pm 0.055$ \\
\hline $2.0 \pm 0.1$ & $0.260 \pm 0.01$ & $1.705 \pm 0.006$ & $1.978 \pm 0.0010^{\circ}$ & $2.128 \pm 0.003$ & $0.423 \pm 0.009$ & $1.100 \pm 0.122$ & $1.850 \pm 0.026$ & $0.750 \pm 0.192$ \\
\hline $2.0 \pm 0.1$ & $0.600 \pm 0.01$ & $1.831 \pm 0.005$ & $1.990 \pm 0.0010^{\circ}$ & $2.307 \pm 0.007$ & $0.476 \pm 0.012$ & $1.492 \pm 0.150$ & $1.343 \pm 0.152$ & $-0.149 \pm 0.258$ \\
\hline $3.5 \pm 0.1$ & $0.020 \pm 0.01$ & $1.775 \pm 0.003$ & $1.990 \pm 0.0010^{\circ}$ & $2.018 \pm 4.7210^{-4}$ & $0.243 \pm 0.003$ & $0.628 \pm 0.062$ & $1.910 \pm 1.0010^{-2}$ & $1.282 \pm 0.072$ \\
\hline $3.5 \pm 0.1$ & $0.340 \pm 0.01$ & $1.805 \pm 0.006$ & $1.999 \pm 0.0010^{\circ}$ & $2.255 \pm 0.007$ & $0.450 \pm 0.013$ & $1.347 \pm 0.109$ & $1.649 \pm 0.144$ & $0.302 \pm 0.253$ \\
\hline $3.5 \pm 0.1$ & $0.500 \pm 0.01$ & $1.850 \pm 0.005$ & $1.998 \pm 0.0010^{\circ}$ & $2.302 \pm 0.007$ & $0.452 \pm 0.013$ & $1.541 \pm 0.104$ & $1.435 \pm 0.148$ & $-0.106 \pm 0.252$ \\
\hline $6.5 \pm 0.1$ & $0.010 \pm 0.01$ & $1.932 \pm 0.001$ & $1.992 \pm 0.0010^{\circ}$ & $2.012 \pm 4.2610^{-4}$ & $0.080 \pm 0.001$ & $1.580 \pm 0.020$ & $1.924 \pm 9.0010^{-3}$ & $0.344 \pm 0.029$ \\
\hline $6.5 \pm 0.1$ & $0.360 \pm 0.01$ & $1.836 \pm 0.006$ & $1.994 \pm 0.0010^{\circ}$ & $2.273 \pm 0.008$ & $0.437 \pm 0.014$ & $1.439 \pm 0.109$ & $1.474 \pm 0.161$ & $0.035 \pm 0.270$ \\
\hline $6.5 \pm 0.1$ & $0.440 \pm 0.01$ & $1.853 \pm 0.005$ & $1.994 \pm 0.0010^{\circ}$ & $2.286 \pm 0.008$ & $0.433 \pm 0.013$ & $1.547 \pm 0.105$ & $1.395 \pm 0.160$ & $-0.152 \pm 0.265$ \\
\hline
\end{tabular}

try can be observed. Therefore, the multifractal parameters show sample-to-sample and time-to-time fluctuations.

As time increases, the multifractal parameters are not similar (Table 1). The value of the Hölder exponent at the box dimension $\alpha_{0}$ is not constant in time; in fact, it increases. It achieves values slightly greater than 2 , reflecting the plume expansion. The entropy dimension coefficient $\alpha_{1}$ does not have the same behaviour and, depending on the experimental $H_{0}$ used, its evolution is different. For $H_{0}=2 \mathrm{~cm}, \alpha_{1}$ has a tendency to decrease as the time increases until a certain point where it increases again to its original value. In the case of $H_{0}=3.5 \mathrm{~cm}$, at the beginning, $\alpha_{1}$ has an increase tendency and at the end decreases. Finally, for $H_{0}=6.5 \mathrm{~cm}$, the behaviour of $\alpha_{1}$ is similar to the former case but does not decrease at the end, keeping a constant value.

There are two parameters which are deduced from the graphs of Fig. 6. The first one is the width of the curves (or variation of the Hölder exponent), which is defined as $\Delta \alpha=\alpha_{\max }-\alpha_{\min }$, where $\alpha_{\min }$ is the first value of the Hölder exponent and $\alpha_{\max }$ is the last one (as shown in Fig. 3). This parameter quantifies the width of the multifractal spectrum and indicates the overall variability. We observe that the multifractal spectrum presents different amplitudes for different times as commented earlier.

The second parameter is defined as $\Delta f=f\left(\alpha_{\max }\right)-f\left(\alpha_{\min }\right)$, which is the height of the graphs in Fig. 6 (see Fig. 3 ). This magnitude represents the symmetry of the curve and tends to zero if the curve is more symmetric. The differences between $f\left(\alpha_{\min }\right)$ and $f\left(\alpha_{\max }\right)$ are not constant in time: at the beginning, there is not a clear trend and later they decrease until a negative value (Table 1 ), as commented earlier.

In general, $\Delta \alpha$ increases with time, implying an increase in overall variability in space. This reflects that as the plume evolves over time, the darker areas are found closer to the plume's axis and the borders show lighter greys increasing the variability of grey values comparing to the first frame of the plume. 


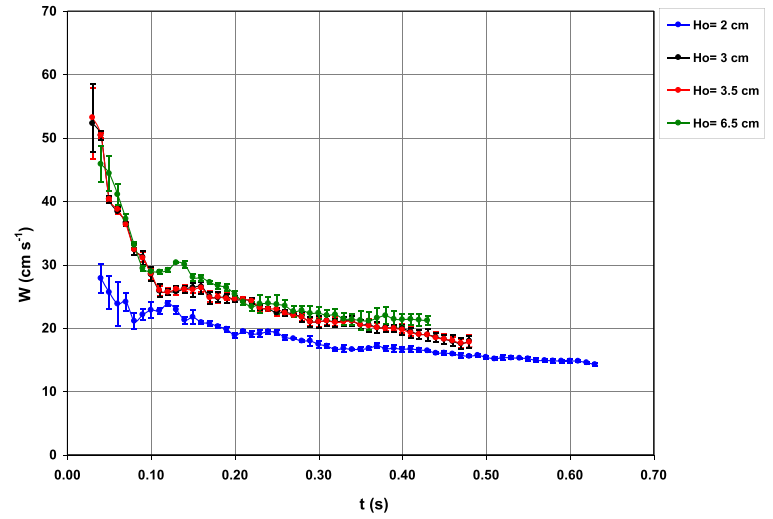

Fig. 7. Time evolution of the axial velocity. We show the vertical velocity $W$ for experiments with $A=0.01$ at different $H_{o}$ values of height.

In the $\Delta f$ case, as said earlier, $\Delta f$ decreases with time achieving negative values at the end. This implies an evolution in the spectrum symmetry along the plume evolution. At the beginning, the highest values (lighter greys) are driving the scaling behaviour and at the end are the darker areas that have a higher importance.

\subsection{Comparison between multifractal parameters and axial velocity}

The axial or vertical velocity $W$ of the plume is an important magnitude (Section 2.2) which has been measured in frames from all different experiments. Fig. 7 shows the time evolution of the vertical speed for experiments with the same Atwood number $(A=0.01)$ and different heights $H_{0}$. As it is observed, initially, the plume presents a great axial velocity. Later, the vertical velocity decreases monotonically and seems to approach an asymptotic value (approximately $15 \mathrm{~cm} \mathrm{~s}^{-1}$ for $H_{o}=2 \mathrm{~cm}, 18 \mathrm{~cm} \mathrm{~s}^{-1}$ for $H_{o}=3.5 \mathrm{~cm}$ and $21 \mathrm{~cm} \mathrm{~s}^{-1}$ for $H_{0}=6.5 \mathrm{~cm}$ ). This time behaviour is similar for all the heights $\mathrm{Ho}$ although the axial velocity and its asymptotic value are greater when $H_{0}$ is greater. The main decrease in the vertical velocity has been realized before reaching the jet length $\left(L_{J}\right.$ ranges between 6 and $\left.7 \mathrm{~cm}\right)$ at approximately $0.30 \mathrm{~s}$. This momentum length scale is reached earlier in time as the height $H_{0}$ is greater. For example, if $H_{0}$ is $2 \mathrm{~cm}, L_{J}$ is reached in $0.38 \mathrm{~s}$ and if $H_{0}$ is $6.5 \mathrm{~cm}$, in $0.28 \mathrm{~s}$. This means that the buoyancy effect dominates the plume behaviour earlier in time as the height $H_{0}$ is greater. After reaching the jet length, the plume goes on to its asymptotic speed.

For a deeper study into the relation between the multifractal parameters deduced earlier (Table 1) and the vertical velocity of the plume, several plots have been made to analyse the evolution of these parameters with axial velocity. Fig. 8 shows the parameter $\alpha_{1}$ versus the axial velocity for the analysed experiments. We observe that the curve presents an inverted U-shape at $H_{0}=2 \mathrm{~cm}$, which disappears at higher Ho. Therefore, this curve achieves a maximum value at different velocities, depending on $H_{0}$. In case of $H_{0}=2 \mathrm{~cm}$, the maximum $\alpha_{1}$ value is achieved at $W$ of approximately $16 \mathrm{~cm} \mathrm{~s}^{-1}$ and when $H_{0}=3.5 \mathrm{~cm}$, it is achieved at $22 \mathrm{~cm} \mathrm{~s}^{-1}$. This implies that the maximum entropy was achieved earlier in the former case. For $H_{0}=6.5 \mathrm{~cm}$, the maximum entropy obtained is lower as the plume arrived the end of the studied frame much earlier, as it can be seen in the axial velocities' values and the number of frames analysed.

On the other hand, in the case of $H_{0}=2 \mathrm{~cm}$, when the entropy diminishes, the axial velocity varies very little and the values are smaller, indicating the interval where the axial velocity trends to a limit as seen in Fig. 7.

Fig. 9 represents the symmetry of the spectrum, $\Delta f$, versus the vertical velocity for all the experiments. We observe that $\Delta f$ of

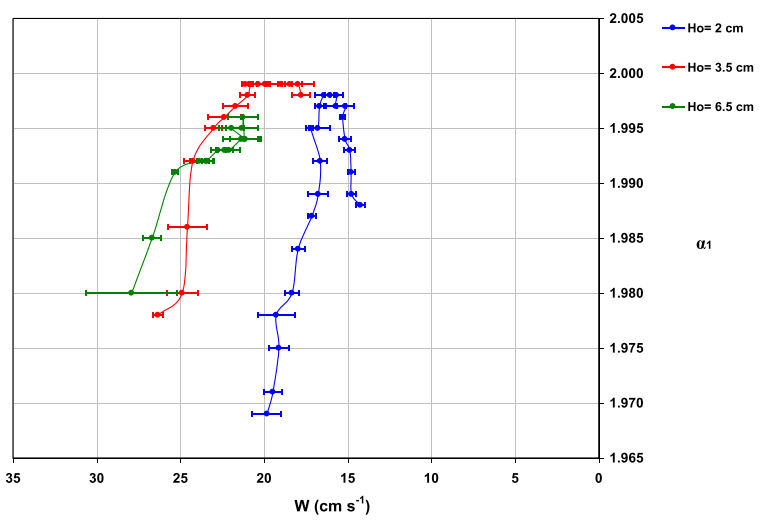

Fig. 8. The Hölder exponent at $q=1$ or $\alpha_{1}$ versus the vertical velocity $W$. The experimental conditions are $A=0.01$ and different values of the height $H_{o}$.

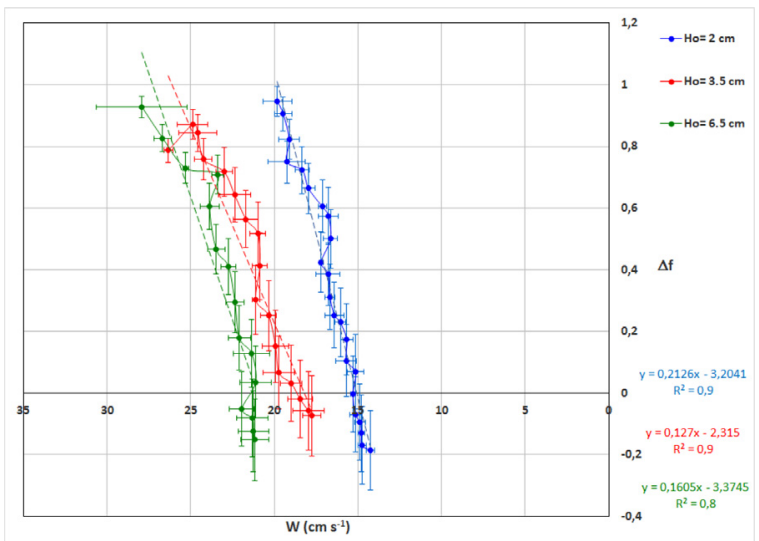

Fig. 9. The height of the spectrum $\Delta f=\mathrm{f}\left(\alpha_{\max }\right)-f\left(\alpha_{\min }\right)$ versus the vertical velocity $W$. The experimental conditions are $A=0.01$ and different values of the height $H_{0}$.

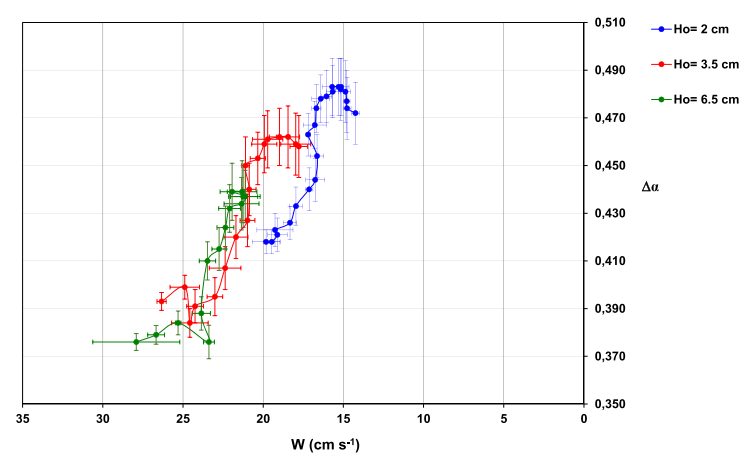

Fig. 10. The width of the spectrum $\Delta \alpha=\alpha_{\max }-\alpha_{\min }$ versus the vertical velocity $W$. The experimental conditions are $A=0.01$ and different values of the height $\mathrm{H}_{0}$.

the spectrum always decreases as the velocity decreases, that is, as time goes on for all the experiments. Then, we deduce that the curve is more symmetric as time goes on and the speed decreases until a certain moment where $\Delta f$ is null. The curve is symmetric when $\Delta f$ is zero and then there is no scaling domination of small values or large ones. This occurs at earlier times and at greater velocities as the height $H_{0}$ grows. Therefore, the spectrum reaches the point of symmetry earlier in time if $H_{O}$ is greater. After this point, the curve becomes slightly asymmetric to the left; that is, large values dominate the scaling. In all the experiments, the $\Delta f$ variation versus axial velocity shows a statistically significant linear trend. 


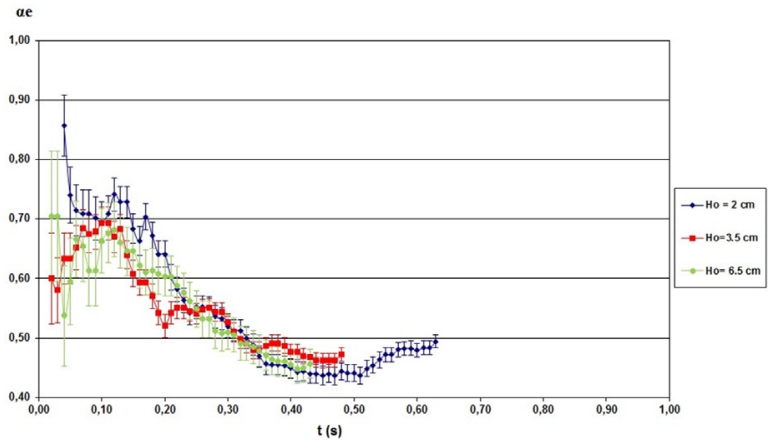

Fig. 11. Time evolution of the entrainment coefficient $\alpha_{e}$. The experimental conditions are $A=0.01$ at different values of the source height $H_{0}$.

Fig. 10 shows the width of the multifractal spectrum, $\Delta \alpha$, versus the vertical velocity for all the experiments. With this parameter, we study the overall complexity, higher is this amplitude more complex is the distribution of the measure studied.

We observe that the curve presents an S-shape at $H_{0}=2 \mathrm{~cm}$, which disappears at higher $H_{0}$, becoming almost linear. In case of $H_{0}=2 \mathrm{~cm}$, the maximum $\Delta \alpha$ value is achieved at approximately $16 \mathrm{~cm} \mathrm{~s}^{-1}$ and when $H_{0}=3.5 \mathrm{~cm}$ it is achieved at $22 \mathrm{~cm} \mathrm{~s}^{-1}$. This implies that the maximum amplitude reached earlier in the former case and these maxima coincide with the ones achieved in entropy, as it can be seen in Fig. 8 . At $H_{0}=6.5 \mathrm{~cm}$, the maximum amplitude obtained is slightly lower. However, the trend showed in all the frames are very similar to the case of $H_{0}=3.5 \mathrm{~cm}$.

Similar behaviour is observed if we compare Figs. 8 and 10. In the case of $H_{0}=2 \mathrm{~cm}$, when the amplitude diminishes the axial velocity varies very little and the values are smaller, indicating the interval where the axial velocity trends to a limit. In the other two cases, this cannot be observed as the plume reaches the end of the frame sooner.

\subsection{Comparison between multifractal parameters and entrainment coefficient}

We have just demonstrated that the used multifractal parameters are able to describe the evolution of the plume at different experimental conditions. Therefore, we can use these parameters to analyse the rate of entrainment, which is represented by the entrainment coefficient $\left(\alpha_{e}\right)$. Other authors have done similar studies. For example, Qianqian et al. have analysed the variation of the fractal dimension of dye plume images at different source distances [42].

The entrainment coefficient $\alpha_{e}$ is not a constant and it presents time and spatial variations [43-46]. Fig. 11 shows the decrease in time of $\alpha_{e}$ for different experimental conditions. At early times or in near-source regions, the values of $\alpha_{e}$ are greater and depend clearly on $H_{0}$. Close to the plume source, the concentration field fluctuates more in both space and in time and the entrainment coefficient has greater values.

Later, in time or at far downstream regions, the entrainment coefficient tends to a smaller value (approximately 0.45 ) in all studied cases. The concentration field is more homogeneous because the dye concentration spreads widely due to relative diffusion and then the coefficient $\alpha_{e}$ decreases. This global time evolution is similar to other authors' results [47].

Fig. 12 shows the entropy dimension $\left(\alpha_{1}\right)$ versus the entrainment coefficient $\left(\alpha_{e}\right)$ for different experimental conditions. We verify that when $\alpha_{e}$ diminishes through time, $\alpha_{1}$ increases. In case of $H_{0}=2 \mathrm{~cm}$, this behaviour is more evident. We deduce an approximately linear relationship between $\alpha_{1}$ and $\alpha_{e}$. This behaviour is confirmed by the $r$-squared coefficient of the corresponding linear

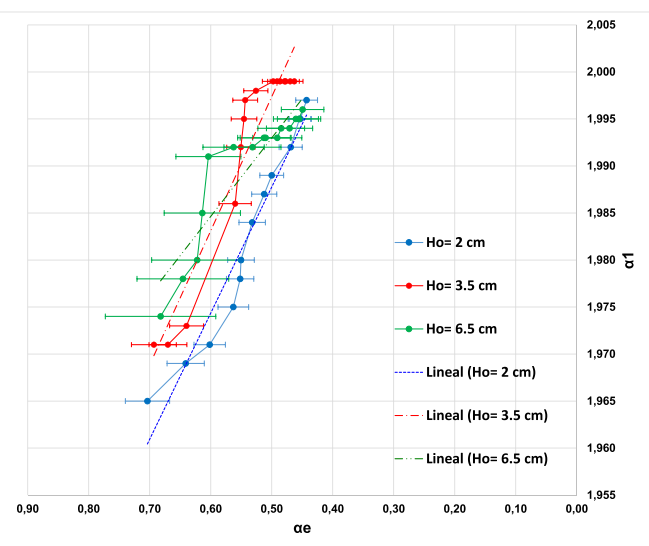

Fig. 12. The Hölder exponent $\alpha_{1}$ versus the entrainment coefficient $\alpha_{e}$ (inverted axis). The experimental conditions are $A=0.01$ and different values of the height $\mathrm{H}_{0}$.

fits that are the following:

$\begin{array}{lll}H o=2 \mathrm{~cm}: & \alpha_{1}=-0.13 \cdot \alpha_{e}+2.06 & r^{2}=0.95 \\ H o=3.5 \mathrm{~cm}: & \alpha_{1}=-0.14 \cdot \alpha_{e}+2.07 & r^{2}=0.92 . \\ H o=6.5 \mathrm{~cm}: & \alpha_{1}=-0.08 \cdot \alpha_{e}+2.04 & r^{2}=0.84\end{array}$

We also observe that the linear fit is less robust as the source distance $H_{O}$ increases and, therefore, the best fit is for $H_{0}=2 \mathrm{~cm}$. That is, the increase in the momentum flux (i.e., plume behaviour more such a jet) makes the linear fit not so good.

Considering Fig.s 11 and 12, the entropy dimension $\alpha_{1}$ is lower close to the source where the entrainment coefficient is greater. $\alpha_{1}$ is greater for far downstream regions where the plume spreads and the entrainment coefficient decreases. As we describe in Section 2.2, that a $\alpha_{1}$ value close to 1.0 characterizes a system uniformly distributed throughout all scales. Therefore, there is a relation between the entrainment coefficient and the not uniform concentration in our range of scales. Furthermore, as the coefficient $\alpha_{e}$ decreases, this non-uniformity increases because $\alpha_{1}$ increases (Fig. 12). This means that in nearly-source regions (i.e., at an early time), the concentration field is less homogeneous and the entrainment coefficient is greater.

\section{Conclusions}

The main objective of this paper was to apply a multifractal analysis (MFA) for studying the behaviour of a turbulent axisymmetric plume under different experimental conditions and its relation with the axial velocity. This was done by analysing the series of images that describe the evolution of the plume.

The MFA on each grey image, applying the box gliding method, revealed a multiscaling nature from 1 to 196 pixels side length of the gliding box. The partition function and the mass exponent function estimated are characteristics of a multifractal measure with a non-fractal support.

Measurements of the vertical velocity of a plume, in intervals of $0.01 \mathrm{~s}$, have shown that the axial velocity increases with height $H_{o}$ and decreases monotonically with time, as previous reported in other studies. After certain time, the vertical velocity trends asymptotically to the same value regardless of the height.

On the other hand, several parameters derived from the MFA were estimated at different times and experimental height $H_{0}$. The results showed an evolution of $\alpha_{1}, \Delta \alpha$ and $\Delta f$ that can describe the evolution of the plume in the cases studied $\left(H_{0}=2,3.5\right.$, and $6.5 \mathrm{~cm}$ and Atwood number equal to 0.01 ). Therefore, the time evolution of the plume (its spreading) and the effect of momentum flux can be approached by means of the multifractal param- 
eters $\alpha_{1}, \Delta \alpha$, and $\Delta f$. This is a key conclusion because, from this point on, we can use the multifractal method and its parameters as markers of plume behaviour. At the same time, the $f(\alpha)-\alpha$ curves estimated are similar to the ones obtained from the energy dissipation field, which is caused by the velocity gradients at the viscous scales [48]. The similar $f(\alpha)-\alpha$ curves might imply that the plume structure in turbulent convection is formed to maximize the entropy of the structure [48,49].

Additionally, we plotted each multifractal parameter with the axial velocity obtained in each photogram by marking the similar evolution they present in all the source distances used in the experiment. However, the curves describing the experiment with $H_{0}=2 \mathrm{~cm}$ were always separate from the other two, mainly due to the lower values present in the axial velocity.

We have also showed the time decrease of the entrainment coefficient $\left(\alpha_{e}\right)$ for different experimental conditions. We have demonstrated the different behaviour of $\alpha_{e}$ depending on whether we are near or far from the plume source. Finally, we have analysed the linear relation between the entropy dimension $\left(\alpha_{1}\right)$ and the entrainment coefficient $\left(\alpha_{e}\right)$. We have verified that when $\alpha_{e}$ diminishes through time, $\alpha_{1}$ increases and, therefore, is related to the non-homogeneity of the concentration field. In conclusion, we are characterizing the rate of entrainment by a multifractal parameter that we have demonstrated for the first time.

Further research is in progress using the multifractal approach to analyse the effect of buoyancy flux and to compare it with the effect of momentum flux we have just studied. Another goal will be to study the local space behaviour of the entrainment constant.

\section{Acknowledgments}

Authors would like to thank all the referees for their help and valuable remarks. We would also like to thank the publishing team at Chaos, Solitons \& Fractals for all their assistance and facilities. This work was supported by the European Community (PITN-GA2008-214919); and by MINECO (Ministry of Economy and Competitiveness), contract No. MTM2015-63914-P). Finally, authors gratefully acknowledge the technical support of the Instituto Pluridisciplinar (University Complutense of Madrid) along this experimental work and the help from ERCOFTAC (SIG 14).

\section{References}

[1] Morton BR, Taylor GI, Turner JS. Turbulent gravitational convection from maintained and instantaneous sources. P Roy Soc Lon Ser-A 1956;234:1-23. doi:10. 1098/rspa.1956.0011.

[2] List EJ. Turbulent jets and plumes. Annu Rev Fluid Mech 1982;14:189-212. doi:10.1146/annurev.fl.14.010182.001201.

[3] DePaolo DJ, Stolper EM. Models of Hawaiian volcano growth and plume structure: Implications of results from the Hawaii scientific drilling project. J Geophys Res 1996;101(B5):11643-54. doi:10.1029/96JB00070.

[4] Harvey DC, Gaonach H, Lovejoy S, Stix J. Multifractal characterization of remotely sensed volcanic features: a case study from Kilauea volcano. Hawaii. Fractals 2002;10:265-74. doi:10.1142/S0218348X02001191.

[5] Stankiewicz J, de Wit MJ. Fractal river networks of Southern Africa. S Afr J Geol 2005;108(3):333-44. doi:10.2113/108.3.333

[6] Claps P, Oliveto G. Reexamining the determination of the fractal dimension of river networks. Water Resour Res 1996;32:3123-35. doi:10.1029/96WR01942.

[7] Lovejoy S, Mandelbrot BB. Fractal properties of rain and a fractal model. Tellus A 1985;37(3):209-32. doi:10.1111/j.1600-0870.1985.tb00423.x.

[8] Lovejoy S. Area-perimeter relation for rain and cloud areas. Science 1982;216(4542):185-7. doi:10.1126/science.216.4542.185

[9] Csanady GT. Turbulent diffusion in the environment. 1st ed. Dordrecht: Reidel Publishing Company; 1973. doi:10.1007/978-94-010-2527-0.

[10] Fackrell JE, Robins AG. Concentration fluctuations and fluxes in plumes from a point source in a turbulent boundary layer. J Fluid Mech 1982;117:1-26. doi:10. 1017/S0022112082001499.

[11] Mylne KR, Mason PJ. Concentration fluctuation measurements in a dispersing plume at a range up to $1000 \mathrm{~m}$. Q J Roy Meteor Soc 1991;117:177-208. doi:10. 1002/qj.49711749709.

[12] Sreenivasan KR. Fractal and multifractals in fluid turbulence. Annu Rev Fluid Mech 1991;23:539-600. doi:10.1146/annurev.fl.23.010191.002543.
[13] Hentschel HE, Procaccia I. Relative diffusion in turbulent media: the fractal dimension of clouds. Phys Rev A 1984;29:1461-71. doi:10.1103/PhysRevA.29. 1461.

[14] Prasad RR, Sreenivasan KR. The measurement and interpretation of fractal dimensions of surfaces in turbulent flows. Phys Fluids A-Fluid 1990;2:792-807. doi:10.1063/1.857733.

[15] Sreenivasan KR, Meneveau C. The fractal facets of turbulence. J Fluid Mech 1986;173:357-86. doi:10.1017/S0022112086001209.

[16] Sreenivasan KR, Ramshankar R, Meneveau C. Mixing, entrainment and fractal dimensions of surfaces in turbulent flows. P Roy Soc A-Math Phy 1989;421:79108. doi:10.1098/rspa.1989.0004.

[17] Sykes RI, Gabruk RS. Fractal representation of turbulent dispersing plumes. J Appl Meteorol 1994;33:721-32. doi:10.1175/1520-0450(1994)033.

[18] Lane-Serff GF. Investigation of the fractal structure of jets and plumes. J Fluid Mech 1993;249:521-34. doi:10.1017/S0022112093001272.

[19] Frisch U, Parisi G. Fully developed turbulence and intermittency. In: Ghil M Benzi R, Parisi G, editors. Turbulence and predictability in geophysical fluid dynamics and climate dynamics. North-Holland: Amsterdam; 1985. p. 71-88.

[20] Meneveau C, Sreenivasan KR. Simple multifractal cascade model for fully developed turbulence. Phys Rev Lett 1987;59:1424-7. doi:10.1103/PhysRevLett.59. 1424.

[21] Meneveau C, Sreenivasan KR. Interface dimension in intermittent turbulence. Phys Rev A 1990;41:2246-8. doi:10.1103/PhysRevA.41.2246.

[22] Prasad RR, Meneveau C, Sreenivasan KR. Multifractal nature of the dissipation field of passive scalars in fully turbulent flows. Phys Rev Lett 1988;61(1):74-7. doi:10.1103/PhysRevLett.61.74.

[23] Puthenveettil BA, Anathakrishna G, Arakeri JH. The multifractal nature of plume structure in high-Rayleigh-number convection. J Fluid Mech 2005;526:245-56. doi:10.1017/S0022112004002897.

[24] López González-Nieto P. Dissertation. Complutense University of Madrid (UCM): 2004.

[25] López P, Cano JL, Redondo JM. An experimental model of mixing processes generated by an array of top-heavy turbulent plumes. Il Nuovo Cimento 2008;31C(5-6):679-98. doi:10.1393/ncc/i2009-10330-y.

[26] Turner JS. Turbulent entrainment: the development of the entrainment assumption, and its application to geophysical flows. J Fluid Mech 1986;173:43171. doi:10.1017/S0022112086001222.

[27] Scase MM, Caulfield CP, Linden PF, Dalziel SB. Local implications for selfsimilar turbulent plume models. J Fluid Mech 2007;575:257-65. doi:10.1017| S0022112007004740.

[28] Morton BR. Forced plumes. J Fluid Mech 1959;5(1):151-63. doi:10.1017/ S002211205900012X

[29] Rasband W. Image 1.36, USA: National Institutes of Health; 2006. 153 p.

[30] Elliott TR, Heck RJ. A comparison of 2D vs. 3D thresholding of X-ray CT imagery. Can J Soil Sci 2007;87(4):405-12. doi:10.4141/CJSS06017.

[31] Grau J, Méndez V, Tarquis AM, Díaz MC, Saa A. Comparison of gliding box and box-counting methods in soil image analysis. Geoderma 2006;134(3):349-59. doi:10.1016/j.geoderma.2006.03.009.

[32] Plotnick RE, Gardner RH, Hargrove WW, Prestegaard K, Perlmutter M. Lacunarity analysis: a general technique for the analysis of spatial patterns. Phys Rev E 1996;53:5461-8. doi:10.1103/PhysRevE.53.5461.

[33] Cheng 0 . The gliding box method for multifractal modelling. Comput Geosci 1999;25:1073-9.

[34] Aguado PL, Del Monte JP, Moratiel R, Tarquis AM. Spatial characterization of landscapes through multifractal analysis of DEM. Sci World J 2014;2014:1-9. doi: $10.1155 / 2014 / 563038$

[35] Tarquis AM, Platonov A, Matulka A, Grau J, Sekula E, Díez M, et al. Application of multifractal analysis to the study of SAR features and oil spills on the ocean surface. Nonlinear Proc Geoph 2014;21:439-50. doi:10.5194/npg-21-439-2014.

[36] Tarquis AM, Losada JC, Benito R, Borondo F. Multifractal analysis of the Tori destruction in a molecular Hamiltonian system. Phys Rev E 2001;65:126-213. doi:10.1103/PhysRevE.65.016213.

[37] Tarquis AM, Giménez D, Saa A, Díaz MC, Gascó JM. Scaling and multiscaling of soil pore systems determined by image analysis. In: Pachepsky Y, Radcliffe DE, Magdi Selim H, editors. Scaling methods in soil physics. Florida: CRC Press; 2003. p. 19-33.

[38] Shannon CE, Weaver W. The mathematical theory of communication. 10th ed. Urbana: The University of Illinois Press; 1964.

[39] Andraud C, Beghdadi A, Lafait J. Entropic analysis of random morphologies Physica A 1994;207:208-12. doi:10.1016/0378-4371(94)90374-3.

[40] Gouyet JF. Physics and fractal structures. 1st ed. New York: Springer-Verlag Inc; 1996.

[41] Feder J. Multifractal measures. In: Feder J, editor. Fractals. New York: Springer US; 1988. p. 66-103. doi:10.1007/978-1-4899-2124-6.

[42] Shao QQ Sekine D, Tsukahara T, Kawaguchi Y. Inverse source locating method based on graphical analysis of dye plume images in a turbulent flow. Open J Fluid Dyn 2016:6:343-60. doi:10.4236/ojfd.2016.64026.

[43] Van Reeuwijk M, Craske J. Energy-consistent entrainment relations for jets and plumes. J Fluid Mech 2015;782:333-55. doi:10.1017/jfm.2015.534.

[44] Craske J, Van Reeuwijk M. Energy dispersion in turbulent jets. Part 1. direct simulation of steady and unstedy jets. J Fluid Mech 2015;763:500-37. doi:10. 1017/jfm.2014.640.

[45] Matulka A, López P, Redondo JM, Tarquis A. On the entrainment coefficient in a forced plume: quantitative effects of source parameters. Nonlinear Proc Geoph 2014;21:269-78. doi:10.5194/npg-21-269-2014. 
[46] Ezzamel A, Salizzoni P, Hunt GR. Dynamical variability of axisymmetric buoyant plumes. J Fluid Mech 2015;765:576-611. doi:10.1017/jfm.2014.694.

[47] Krug D, Chung D, Philip J, Marusic I. Global and local aspects of entrainment in temporal plumes. J Fluid Mech 2017;812:222-50. doi:10.1017/jfm.2016.786.
[48] Meneveau C, Sreenivasan KR. The multifractal nature of turbulent energy dissipation. J Fluid Mech 1991;224:429-84. doi:10.1017/s0022112091001830.

[49] Chhabra AB, Meneveau C, Jensen RV, Sreenivasan KR. Direct determination of the $f(\alpha ;)$ singularity spectrum and it application to fully developed turbulence. Phys Rev A 1989;40:5284-94. doi:10.1103/physreva.40.5284. 